\title{
Nano-apatite/polymer composites: mechanical and physicochemical characteristics
}

\author{
Qing Liu* ${ }^{\star \dagger}$, Joost R. de Wijn* and Clemens A. van Blitterswijk*† \\ *Biomaterials Research Group, Leiden University, Professor Bronkhorstlaan 10, Building 57, 3723 MB Bilthoven \\ The Netherlands; ${ }^{\dagger}$ Institute for Biomedical Technology. Twente University, 7500 AE Enschede, The Netherlands
}

Hydrothermally synthesized acicular nano-apatite (Nap) was used as filler to make composites with a polyethylene glycol/poly(butylene terephthalate) (PEG/PBT) block copolymer (Polyactive ${ }^{\mathrm{TM}} 70: 30$ ). The Nap had a particle diameter of 9-25 nm and a length of $80-200 \mathrm{~nm}$. The mechanical properties and the physiochemical characteristics of the composites, such as Young's modulus, swelling degree in water and the calcification behaviour, have been determined. It was found that Nap had a strong ability to promote the calcification of composites when incorporated into Polyactive 70:30, while poly(acrylic acid) (PAA) coating of Nap had an adverse effect on the calcification of composites, presumably due to the formation of complexes between PAA and PEG segments. Nap had a prominent stiffening effect for Polyactive 70:30 in the dry state, but had a poor stiffening effect for composites in an aqueous environment due to the hygroscopic nature and/or the formation of aggregates. PAA coating on Nap had almost no additional effect on the mechanical properties of composites either in the dry state or in an aqueous environment. To reinforce the polymer by Nap, achieving a more homogeneous dispersion of Nap in the polymer matrix and surface modifications to render the powders less hygroscopic appear to be necessary. (C) 1997 Elsevier Science Limited. All rights reserved

Keywords: Composites, calcium apatite, nano-apatite, Polyactive ${ }^{T M}$, polyether-ester, calcification, coating, polyacrylic acid, nanoparticles

Received 2 January 1997; accepted 21 March 1997

Composites, due to the possibility of combining the advantages of different materials, have attracted much attention from material scientists. Because of its biocompatibility and bone bonding ability ${ }^{1-5}$, hydroxyapatite (HA) has been used as a bone substitute material as such, but also as a filler in composites with organic polymers. In these cases, synthetic HA is used in the form of polygonal sintered coarse particles with polycrystalline structure, which have little similarity to natural bone mineral as far as crystal size and shape are concerned. Some researchers have suggested that better osteoconductivity would be achieved if HA had more similarity to bone mineral in composition, crystal structure, crystallinity, crystal size and morphology ${ }^{6-b}$. Hydrothermally synthesized nanoapatite (Nap) is a kind of carbonated apatite which has an acicular or needle-like shape 9 . It has much more similarity to natural bone mineral in the mentioned compositional and morphological aspects and therefore better osteoconductivity is expected. In addition to its similarity to bone mineral, the Nap may possess other special properties due to its submicron size and consequently huge specific surface area. Sincenanopar-

Correspondence to Dr Q. Liu, Department of Biomedical Engineering, Rice University, 6100 South Main Street, Houston, TX 77005, USA. ticles showed quantum size effects in their electronic, optical and chemical properties, much research has been conducted in this area of synthetic materials chemistry $^{10,11}$ and applications in composites with organic polymers ${ }^{10-16}$. When using such nanoparticles to make composites with organic polymers, provided homogeneous dispersion of the nanoparticles can be achieved at the microscopic level, the mechanical properties are expected to be further improved and/or new unexpected features might appear ${ }^{10,11}$.

Polyactive $^{\mathrm{TM}}$, a block copolymer of poly(butylene terephthalate) (PBT) and poly(ethylene glycol) (PEG), is the only bone bonding polymer known at present ${ }^{17}$. The bone bonding properties of the polymer are considered to be derived from the ability of PEG segments to complex calcium ions. Implantation studies with various PEG/PBT copolymers demonstrated that increasing the amount of PEG in the copolymers increases the calcification rate and consequently results in fast bone bonding ${ }^{18}$. However, increasing the soft PEG segments also decreases the mechanical properties and increases the degradation rate of the copolymer, which can be a disadvantageous side effect for some applications ${ }^{19}$. Therefore, some research was performed to increase the calcification rate of the copolymer wilhoul varying the PEG/PBT ratio $^{20,21}$. In an attempt to develop a more bioactive 
and stronger material as bone substitute material, we used hydrothermally synthesized Nap as filler to make composites with Polyactive 70:30, since this Nap is expected to be more bioactive due to its similarity to bone mineral in many aspects ${ }^{9}$.

In making filler-reinforced polymer composites, the interface between the filler particles and polymer matrix will play an important role in determining the ultimate mechanical properties of the composites. To improve the interface of Nap with Polyactive 70:30, poly(acrylic acid) (PAA) was used as coupling agent, since our previous study has shown that PAA is effective in improving the interface of sintered $\mathrm{HA}$ with Polyactive ${ }^{22,23}$ : composites with PAA as coupling agent can maintain much higher mechanical properties when tested in an aqueous environment as compared to composites without PAA coupling agent.

\section{MATERIALS AND METHODS}

\section{Nap}

Nap was hydrothermally synthesized as described elsewhere ${ }^{8}$. To improve the interface of Nap with PEG/ PBT copolymer, PAA was used as coating. For the coating process, $80 \mathrm{~g}$ hydrothermally synthesized Nap was transferred to $1800 \mathrm{ml} 2 \mathrm{mM}$ PAA sodium salt $\left(\mathrm{M}_{\mathrm{w}}=5100\right)$ solution ( $\mathrm{pH}$ adjusted to 6 using $1 \mathrm{M} \mathrm{HCl}$ ) and stirred for $24 \mathrm{~h}$. Then, the $\mathrm{pH}$ of the suspension was brought down to 5 and washed with ethanol to remove unabsorbed PAA. Finally, the Nap was thoroughly washed with acetone. The non-coated Nap underwent the same procedure, omitting PAA from the solution.

\section{Characterization of Nap}

The size and shape of Nap and PAA-coated Nap were characterized by transmission electron microscopy (TEM; Philips 410). The presence of PAA coating on the surface of Nap was determined by an infrared spectrophotometer (IR; Perkin-Elmer 783) using $\mathrm{KBr}$ tablets. The amount of PAA coating was determined by thermal gravimetrical analysis (TGA; Du Pont 990) using a temperature increase rate of $10^{\circ} \mathrm{C} \mathrm{min}^{-1}$.

\section{Composites}

PEG/PBT copolymer (Polyactive 70:30, HC Implants bv, The Netherlands) had a PEG/PBT weight ratio of 70:30, the molecular weight of PEG being 1000. Certain amounts of PAA-coated and non-coated Nap were mixed into a $15 \%(\mathrm{w} / \mathrm{w})$ Polyactive $70: 30$ chloroform solution. After being intensively stirred, the suspension was dropped into a large amount of diethyl ether. The precipitate was dried first in air and then in a vacuum oven at $50^{\circ} \mathrm{C}$. Composite mixtures with 10,25 and 50 wt \% Nap were obtained. After full removal of the ether, the precipitate was chopped into small pieces and used for hot press moulding at $195^{\circ} \mathrm{C}$ and 20 tons of pressure.

\section{Degree of swelling of the composites}

Samples for swelling tests were cut from hot press shects with a size of $1 \times 1 \times 0.2 \mathrm{~cm}^{3}$. The swelling test was carried out in distilled water at room temperature.
The degree of swelling of the composites was calculated according to the following equation:

$$
S w=\left(W_{t}-W_{0}\right) / W_{0}
$$

where $S w$ is the swelling degree at a certain time interval, $W_{t}$ the weight of the tested specimens after immersion in water at time $t$, and $W_{0}$ the weight of the tested specimens at the beginning of testing.

\section{Mechanical testing}

Rectangular sheets of $2 \mathrm{~mm}$ thickness were made by hot press moulding and dumbbell-shaped specimens for mechanical testing were cut from the sheet with a cutting die (ISO R37 type 1 die). The $E$-modulus, tensile strength and elongation at break were determined in a Houndsfield testing machine at a testing specd of $50 \mathrm{~mm} \mathrm{~min} \mathrm{~m}^{-1}$ at room temperature. The mechanical properties were determined in the dry state and after immersion in phosphate-buffered saline (PBS) solution. In order to accurately measure the $E$ modulus, a strain gauge extension meter (Instron) was used. Ten specimens were used for each testing.

\section{In vitro calcification of the composites}

It is generally believed that the in vitro calcification ability of biomaterials has a correlation with the bone bonding ability in vivo. Therefore, we performed an in vitro test in $\mathbf{1 . 5}$ times simulated body fluid (1.5SBF) which has an ionic concentration 1.5 times the standard concentration of $S_{B F}^{24}$. Samples with a size about $1.5 \times 1.5 \mathrm{~cm}^{2}$ were used for the in vitro calcification of the nano-composite. Fach sample of certain composition was put into a polystyrene beaker with $30 \mathrm{ml} 1.5 \mathrm{SBF}$ and kept at $37^{\circ} \mathrm{C}$ in a shaking water bath. At days 3,6 and 9, samples were taken out and carefully washed by distilled water. After drying and sputter coating with carbon, the samples were subjected to scanning electron microscopy observation and energy dispersive X-ray (EDX) determination.

\section{RESULTS}

\section{Characterization of Nap}

The as-synthesized Nap powder particles had an acicular shape with a width of 9-25 $\mathrm{nm}$ and a length of $80-250 \mathrm{~nm}$ (Figure 1). It had a Brunaeur-EmmettTeller specific surface of $60-80 \mathrm{~m}^{2} \mathrm{~g}^{-1}$. The size and shape were not changed by the PAA coating process.

The IR spectra of PAA-coated powder clearly show the existence of PAA on the surface of the particles (Figure 2). The band at $2880 \mathrm{~cm}^{-1}$ indicates the existence of $\mathrm{CH}_{2}$ vibration. The peak at $1720 \mathrm{~cm}^{-1}$ indicates hydrogen bonded $-\mathrm{C}=\mathrm{O}$ stretching due to hydrogen bond formation between the $-\mathrm{C}=\mathrm{O}$ and the $\mathrm{H}-\mathrm{O}-\mathrm{C}$ of the PAA. The peak at $1568 \mathrm{~cm}^{-1}$ indicates the stretch vibration of $-\mathrm{C}=\mathrm{O}$ groups. The band at $1410 \mathrm{~cm}^{-1}$ is from the vibration of $-\mathrm{C}-\mathrm{O}-\mathrm{H}$.

The amount of PAA coating on the surface of Nap powder as determined by TGA is $5.6 \%$ by weight (Figure 3), which is about one to five PAA molecules on $10 \mathrm{~m}^{2}$ of Nap. The weight loss before $100^{\circ} \mathrm{C}$ was due to the evaporation of surface-absorbed water. 


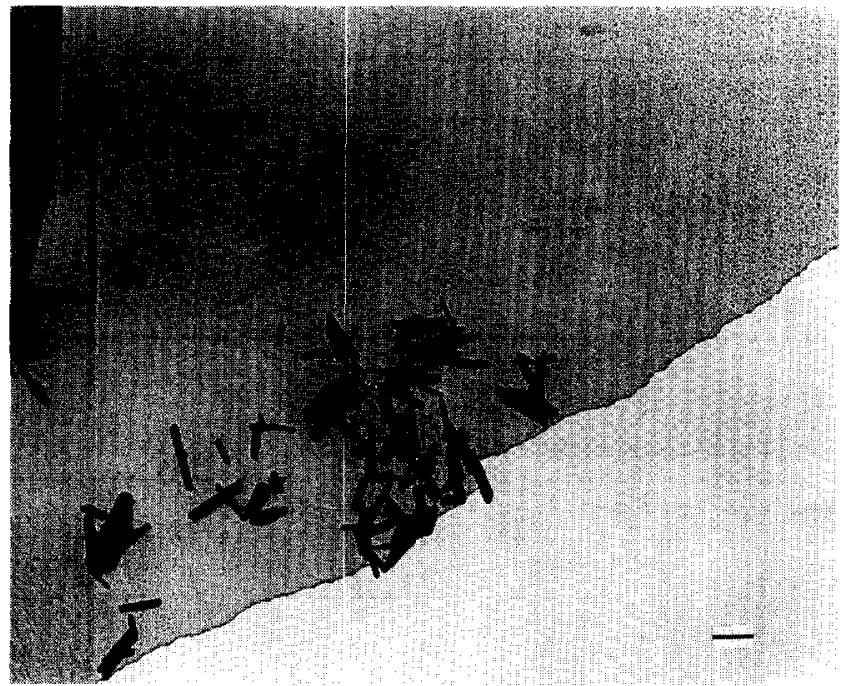

Figure 1 PAA-coated Nap used in this study. The size and shape of the Nap were not changed by the surface treatment with PAA (bar $=100 \mathrm{~nm})$.

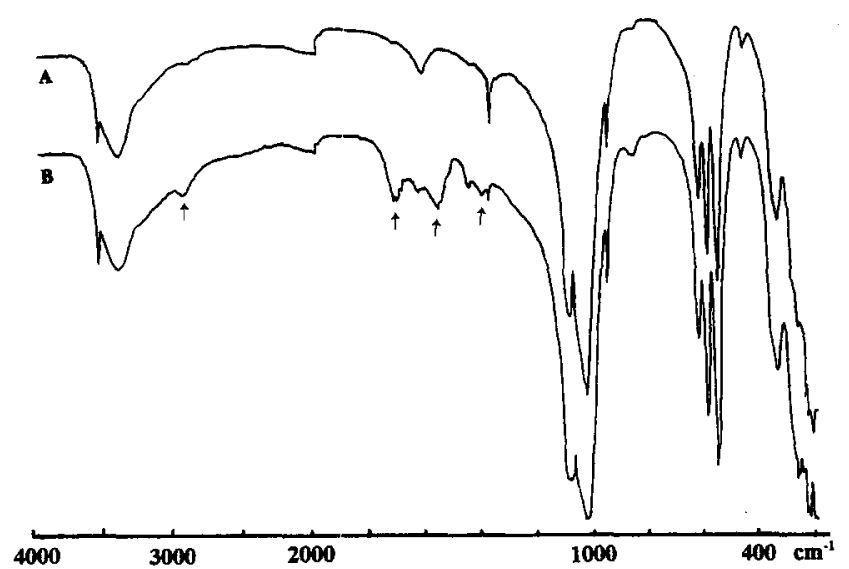

Figure 2 IR spectra of: A, Nap; and B, PAA-Nap used in this study. Note the peaks in spectrum B at 2880,1720, 1568 and $1410 \mathrm{~cm}^{-1}$ indicating the existence of PAA on the Nap.

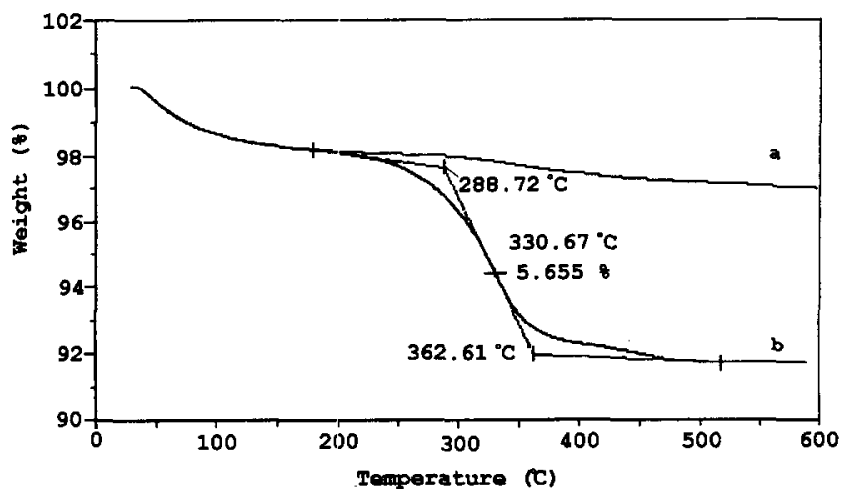

Figure 3 TGA curves of: a, Nap; and b, PAA-Nap which indicate that there was about $5.6 \%$ PAA on the surface of Nap.

\section{Degree of swelling of Nap/polymer composites}

Incorporating Nap into the polymer decreased the uptake of water for the composite, although the uptake was more than would be expected on the basis of proportionality. Swelling gradually reached equilibrium after the samples were soaked in distilled water for $24 \mathrm{~h}$. The PAA-coated Nap composites have a slightly lower degree of swelling as compared to the corresponding composites with non-coated filler (Figure 4).

\section{Mechanical properties of the composites}

The tensile tests showed that, although the elastic modulus of the composites was increased by the incorporation of both non-coated and coated fillers, the tensile strength and elongation at break decreased (Figure 5, Tables 1 and 2). Swelling in water caused a decrease in mechanical properties for all the composites. Although the elastic modulus of $25 \%$ PAA-coated Nap/polymer composites in the wet stage was higher $(18.5 \pm 0.7 \mathrm{MPa})$ than that of noncoated Nap/polymer composites $(15.5 \pm 0.6 \mathrm{MPa})$, generally the effect of PAA coating is negligible with respect to the mechanical properties. An increase of the filler amount decreases the tensile strength and elongation at break. Composites with $50 \%$ filler had very poor mechanical properties. Composites with $50 \%$ PAA-coated filler, probably due to the formation of hydrogen bond complexation between the PEG segment and PAA molecules, were difficult to process into satisfactory samples for mechanical testing.

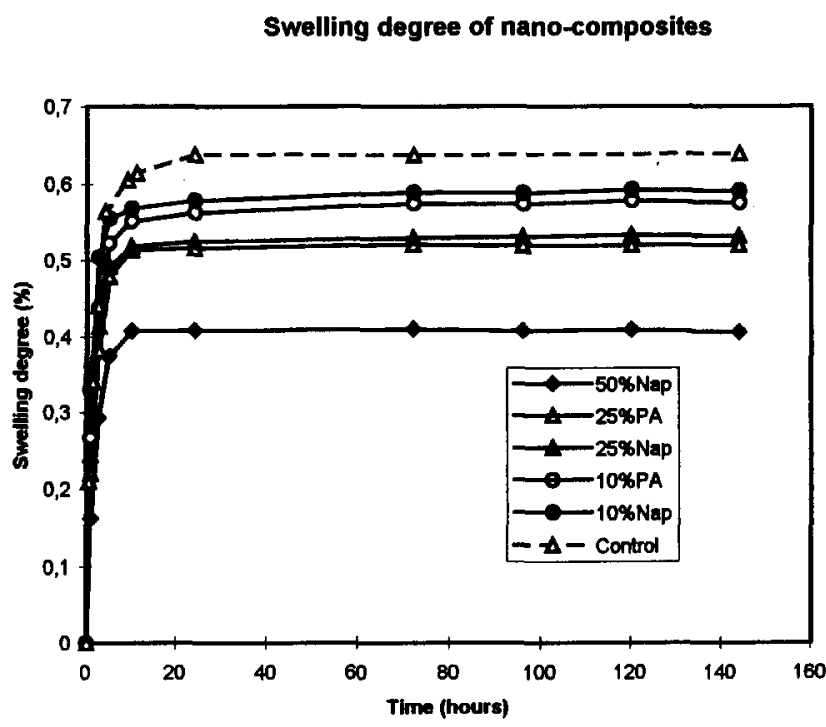

Figure 4 The degree of swelling of the composites. Note that the degree of swelling nearly reached equilibrium after $24 \mathrm{~h}$ immersion in water.

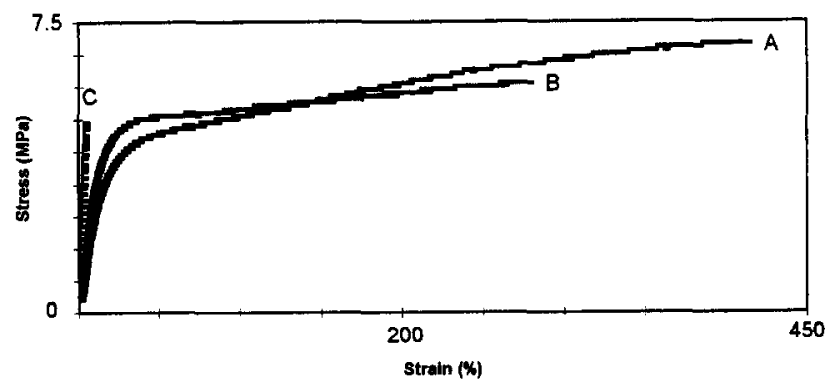

Figure 5 The stress-strain curves of composites in the dry state. The mechanical properties of the composites were affected by the filler in the composites. Curves A, B and C are the tensile test curves of $10 \%, 25 \%$ and $50 \%$ Nap composites in the dry state respectively. 
Table 1 Mechanical properties of the nano-composites in the dry state

\begin{tabular}{|c|c|c|c|c|c|c|}
\hline \multirow[t]{2}{*}{ Filler content $(\%)$} & \multicolumn{2}{|c|}{ E-Modulus (MPa) } & \multicolumn{2}{|c|}{ Tensile strength (MPa) } & \multicolumn{2}{|c|}{ Elongation $(\%)$} \\
\hline & Nap & PAA-Nap & Nap & PAA-Nap & Nap & PAA-Nap \\
\hline 0 & \multicolumn{2}{|c|}{$30.5 \pm 2.1$} & \multicolumn{2}{|c|}{$7.0 \pm 0.2$} & \multicolumn{2}{|c|}{$375 \pm 100$} \\
\hline 10 & $49.1 \pm 1.7$ & $56.0 \pm 6.3$ & $6.8 \pm 0.5$ & $6.5 \pm 0.3$ & $343 \pm 73$ & $354 \pm 29$ \\
\hline 25 & $82.1 \pm 6.3$ & $79.2 \pm 3.3$ & $5.8 \pm 0.2$ & $6.0 \pm 0.3$ & $270 \pm 16$ & $137 \pm 60$ \\
\hline 50 & $242 \pm 27.9$ & n.d. ${ }^{*}$ & $4.8 \pm 0.9$ & n.d. & $8.7 \pm 3$ & n.d. \\
\hline
\end{tabular}

"n.d., not determined.

Table 2 Mechanical properties of the nano-composites after immersion in phosphate-buffered saline for $24 \mathrm{~h}$

\begin{tabular}{|c|c|c|c|c|c|c|}
\hline \multirow[t]{2}{*}{ Filler content $(\%)$} & \multicolumn{2}{|c|}{ E-Modulus (MPa) } & \multicolumn{2}{|c|}{ Tensile strength (MPa) } & \multicolumn{2}{|c|}{ Elongation (\%) } \\
\hline & Nap & PAA-Nap & Nap & PAA-Nap & Nap & PAA-Nap \\
\hline 0 & \multicolumn{2}{|c|}{$7.1 \pm 0.4$} & \multicolumn{2}{|c|}{$4.4 \pm 0.3$} & \multicolumn{2}{|c|}{$87.2 \pm 9.1$} \\
\hline 10 & $17.7 \pm 1.7$ & $16.7 \pm 1.9$ & $3.9 \pm 0.2$ & $3.8 \pm 0.2$ & $91 \pm 16$ & $80 \pm 11$ \\
\hline 25 & $15.5 \pm 0.6$ & $18.5 \pm 0.7$ & $2.9 \pm 0.3$ & $2.8 \pm 0.2$ & $51 \pm 9$ & $51 \pm 8$ \\
\hline 50 & $11.4 \pm 1.0$ & n.d.* & $0.6 \pm 0.1$ & n.d. & $4.8 \pm 0.5$ & n.d. \\
\hline
\end{tabular}

*n.d., not determined.

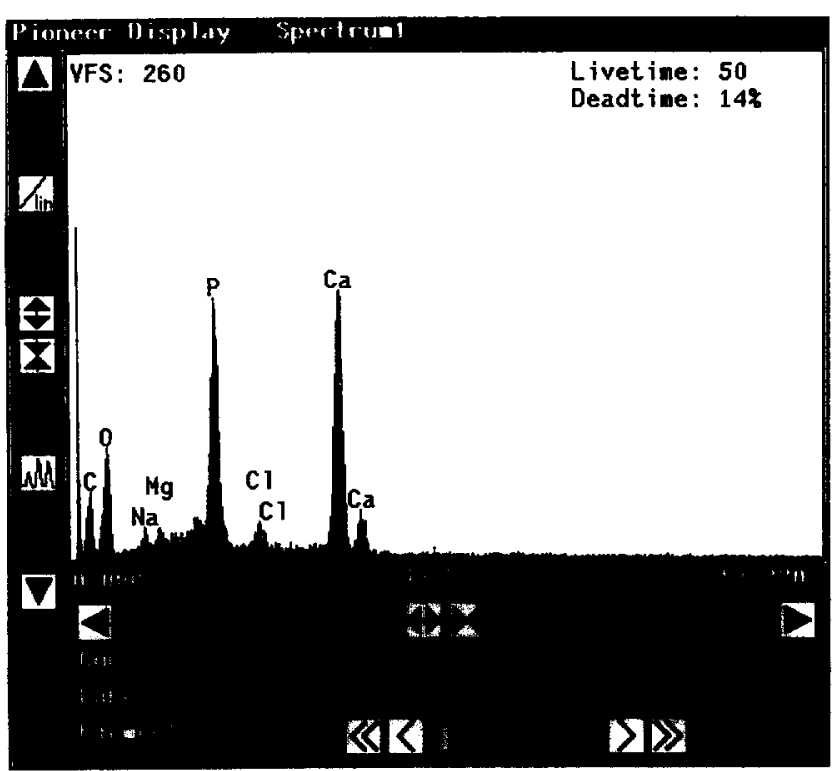

Figure 6 EDX spectrum showing that the mineral layer on top of the $10 \%$ Nap was composed of calcium and phosphate.

\section{The calcification behaviour of the composites}

The calcification experiment showed that the incorporation of non-coated Nap into the polymer matrix significantly promoted calcification of the composites in 1.5SBF (Figures 6 and 7). Composites with untreated Nap filler showed much more calcification in $1.5 \mathrm{SBF}$ as compared to unfilled Polyactive 70:30, in which no calcification was found. Composites with $10 \%$ non-coated Nap filler induced significant amounts of calcium phosphate precipitation on their surfaces (Figure $7 b$ ). The thickness of the calcification layer increased with increase of the soaking time in 1.5SBF. The more Nap present in the composites, the more calcification layer would be obtained in $1.5 \mathrm{SBF}$ (Figure 7d). In contrast, unfilled Polyactive 70:30 failed to induce calcification even after 9 days immersion in 1.5SBF (Figure 7a).

Composites with PAA-coated Nap showed a different calcification behaviour as compared to that of non- coated Nap/polymer composites. While 10\% PAANap composites still showed mineral precipitation from $1.5 \mathrm{SBF}$ after 6 days immersion, the $25 \%$ PAANap/polymer composites could not induce precipitation after 6 days immersion in the same medium.

\section{DISCUSSION}

Generally, using a filler is an effective means to increase the stiffness of a polymer. This is also the case when we use Nap in combination with PEG/PBT copolymer.

When the Nap/polymer composites were tested in the dry state, it seems that the Nap (with or without PAA coating) had a prominent effect on the elastic modulus of the composites (Figure 5). When the Nap filler content was as high as $50 \%$ by weight, the elastic modulus of the composites could be about eight times higher than that of unfilled polymer. However, the decrease in strength indicates that the Nap as filler has no reinforcing effect in terms of tensile strength. Although we have shown that by using PAA as coating $^{22}$, the interface of sintered larger HA particles with Polyactive 70:30 could be distinctly improved, it seems to have less effect on the mechanical properties of the composites in the case of Nap.

Incorporating Nap decreased the degree of swelling of the composites (Figures 4 and 8), although more water was taken up than would be expected on the basis of the assumption that the filler particles do not absorb. In Figure 8, this expected swelling behaviour is plotted together with the observed values. It is obvious that the filler contributes to the water uptake. Extrapolation of the observed degree of swelling values to $100 \mathrm{wt} \%$ filler shows an excess of about $25 \%$ by weight of absorbed water. The hygroscopic nature of the Nap powder was already noticed in the laboratory - extremely dry storage conditions being necessary to prevent the free flowing powder from aggregation and humidification - and is apparently still present in the composites. It is unclear whether the water uptake by the powder takes place through adsorption at the surface of the particles $\left(60-80 \mathrm{~m}^{2}\right)$ or 


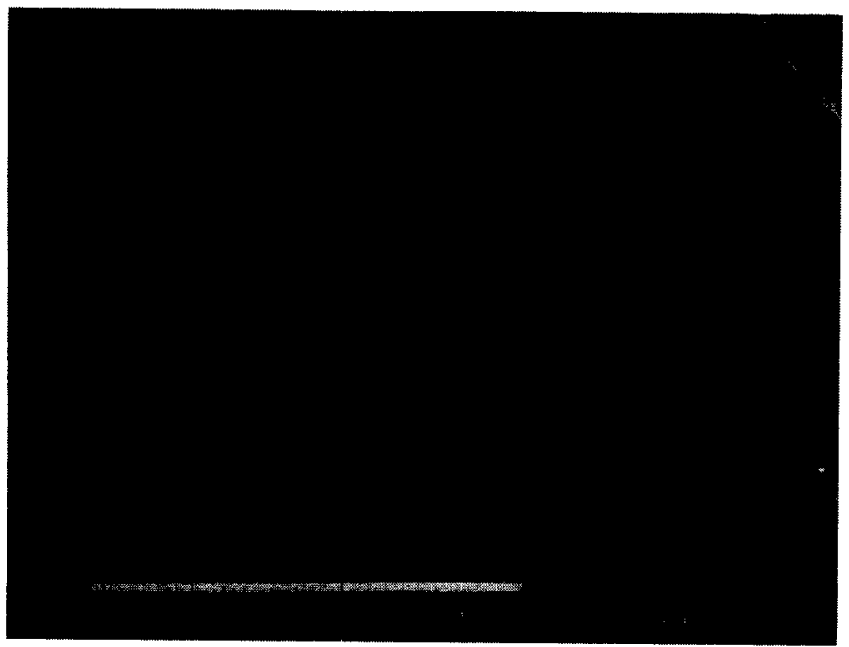

a
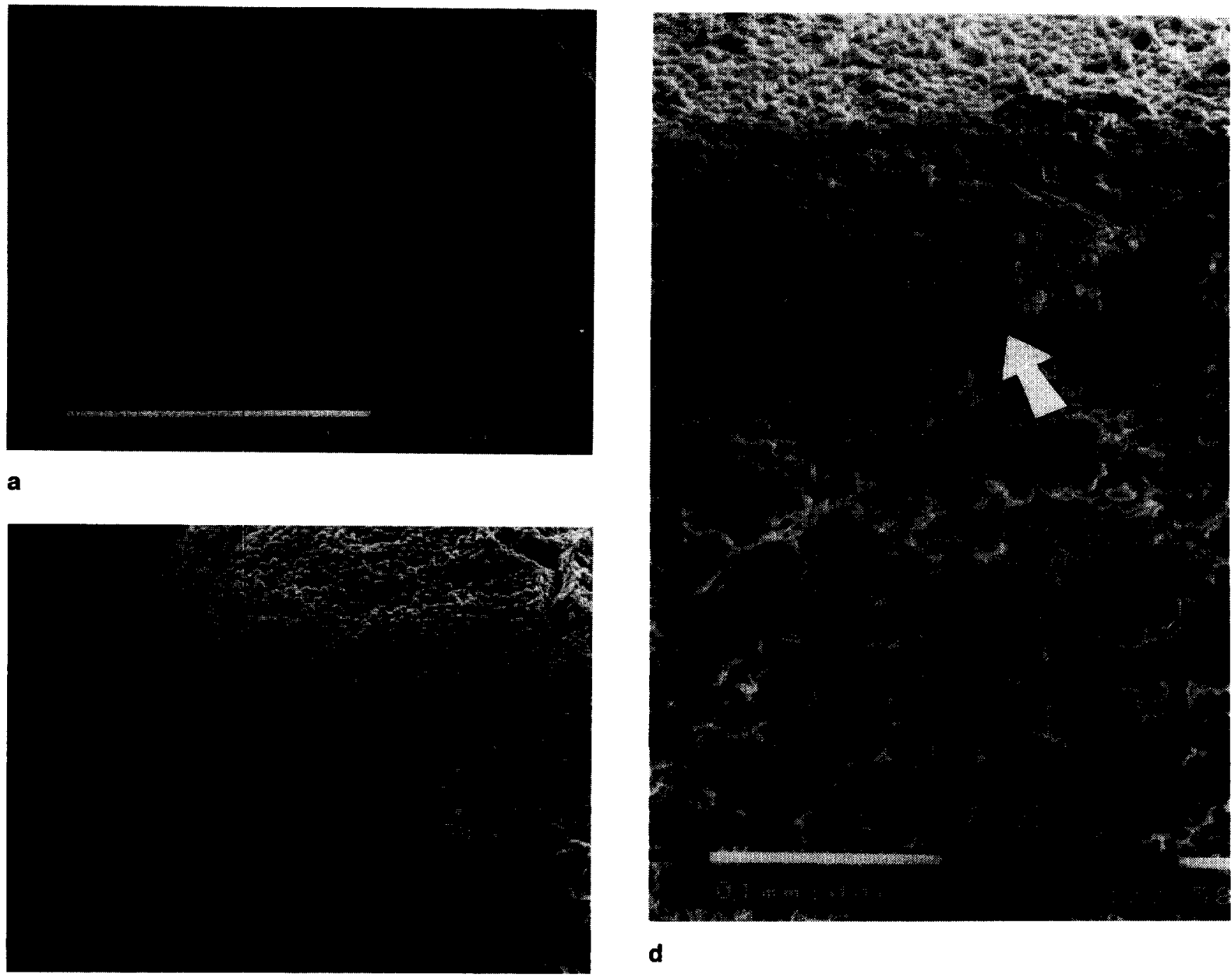

d

b
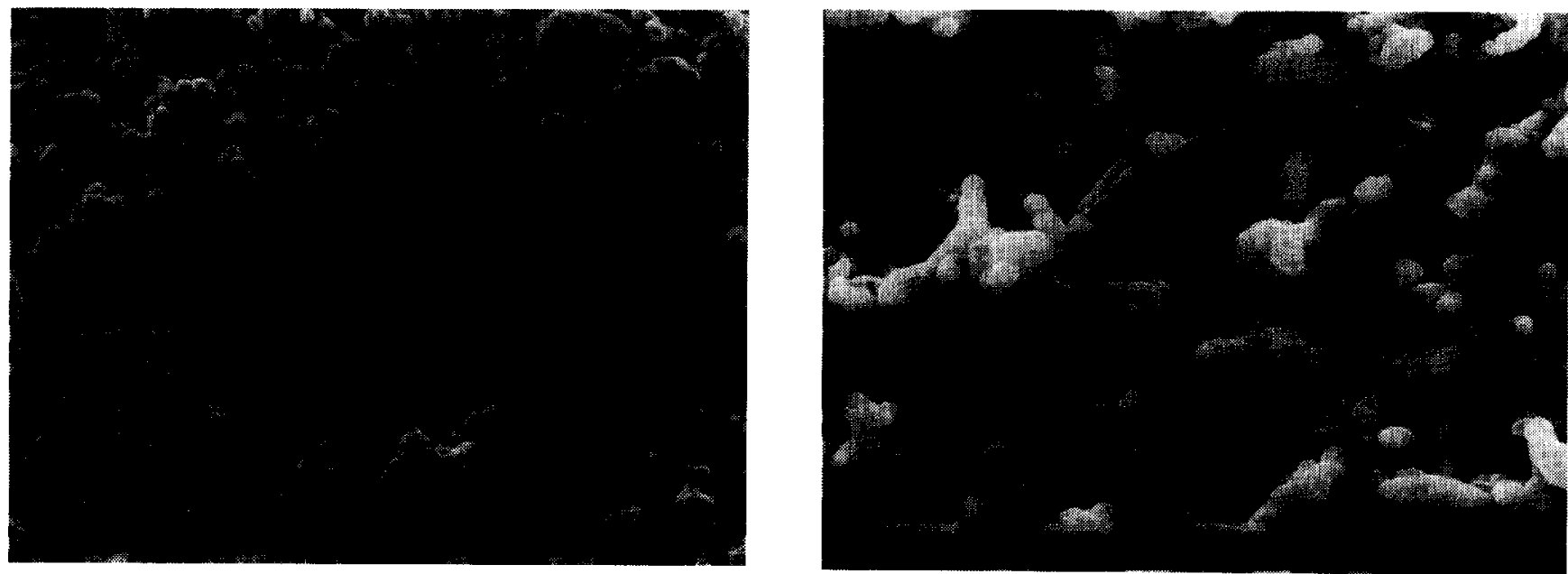

C

Figure 7 a, Polyactive ${ }^{\mathrm{TM}}$ 70:30 samples incubated in 1.5SBF for 3 days. No calcium and phosphate can be detected on the surface of the sample. $b_{\text {. }}$ Composites with $10 \%$ Nap after 3 days immersion in $1.5 \mathrm{SBF}$. The sample was covered by a calcium phosphate layer. c, Calcium phosphate layer on the $25 \%$ Nap composites after 3 days immersion in 1.5SBF. d, A thick calcium phosphate layer was found on the top of 50\% Nap composites after 6 days immersion (cross-section). e, After 6 days immersion in 1.5SEF, 10\% PAA-coated Nap could also induce calcium phosphate precipitation on its surface.

through absorption in capillaries of clusters of the acicular material. The combined water uptake of polymer and filler has a detrimental effect on the mechanical properties of composites, especially if the water uptake occurs at the interface of filler and polymer matrix, which will certainly decrease the adhesion between the two phases. Swelling in PBS caused $50 \%$ filler-containing composites to lose nearly 


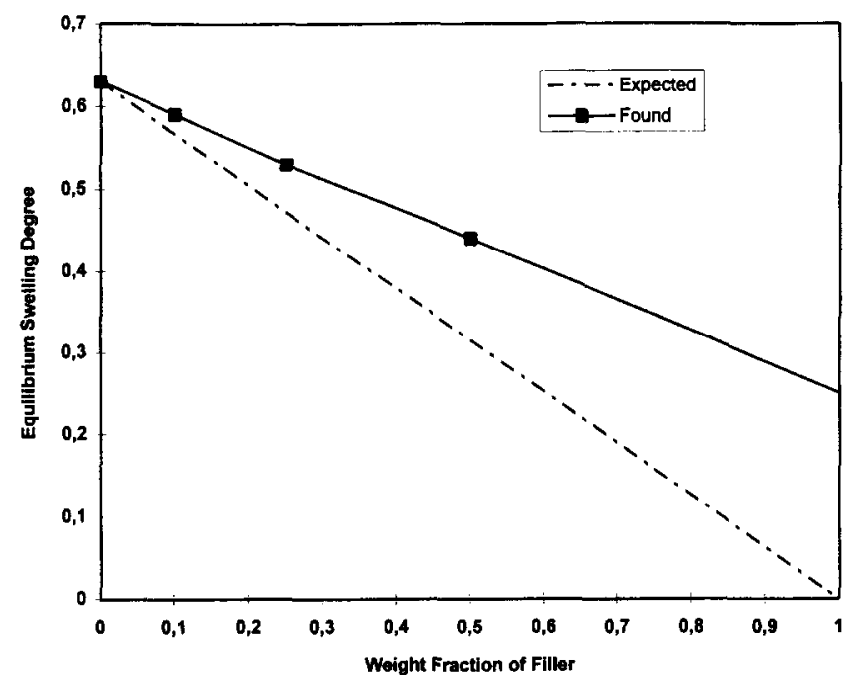

Figure 8 Equilibrium degree of swelling of the Nap composites (without PAA as coupling agent). The dashed line is the expected degree of swelling of Nap composites if only the polymer matrix is considered to take up water Note the difference between the experimental value and the expected value. Apparently the Nap filler contributes to the water uptake of the composites, probably by surface adsorption on Nap or the capillary effect of Nap clusters.

all of their tensile strength (from $4.8 \pm 0.9$ to $0.6 \pm 0.1 \mathrm{MPa}$ ) and at the same time a drastic decrease in elastic modulus occurred (from $242 \pm 27.9$ to $11.4 \pm 1.0 \mathrm{MPa}$ ). Composites with $10 \%$ filler content can maintain relatively reasonable strength $(3.9 \pm 0.2 \mathrm{MPa})$ and elastic modulus $(17.7 \pm 1.7 \mathrm{MPa})$ when compared to those of unfilled polymer ( $4.4 \pm 0.3$ and $7.1 \pm 0.4 \mathrm{MPa}$ respectively). PAA coating seems to have no effect on the mechanical properties of composites, although the coating has a slight effect on the degree of swelling of composites, which can be explained as an indication of complex formation between PAA and PEG segments of the polymer ${ }^{22,23}$ (Figure 8).

The use of PAA as a coating is based on the following principles as discussed in our previous publications ${ }^{22,23}$ : first, PAA can be firmly adsorbed onto the surface of $\mathrm{HA}^{25,26}$; second, PAA can form intermolecular complexes with PEG segments of Polyactive via hydrogen bond or dipole interactions, even when the PEG molecular weight is as low as $1000^{27,28}$. Thus, the use of PAA can significantly improve the interface between $\mathrm{HA}$ particles and Polyactive matrix ${ }^{22,23}$.

In the presenl study, the effect of PAA as a coupling agent can be barely seen from the mechanical testing. The reason for this is mainly due to the poor distribution of Nap in the polymer matrix. It is well known that one of the important factors that determines the mechanical properties of the Nap/polymer composites is the dispersion of the particles in the polymer matrix. It has been indicated that only when the dispersion of the nanoparticles achieves the microscopic level can a significant improvement in mechanical properties be expected $^{10,11}$. Unfortunately, such microscopic level dispersion is very difficult to achieve under the present conditions or in other cases ${ }^{29}$ unless there are strong interactions between filler and polymer matrix $^{30,31}$. In this experiment, agglomeration of the nanoparticles is, besides water absorption, responsible for the observed decrease in tensile strength. It is also a reason why the effect of the PAA coating could not be found in the mechanical properties.

Previous studies have shown that postoperative calcified Polyactive contained needle-shaped carbonated apatite crystals when implanted in vivo. This postoperative calcification probably played an important role for Polyactive in achieving bone bonding $^{32-34}$. Nap added preoperatively to the polymer may promote early bone bonding by accelerating the calcification rate. In fact, we found increased calcification rates in these in vitro experiments.

In this experiment, Polyactive 70:30, for which calcification has been reported both in vitro and in vivo ${ }^{17-21,32-34}$, failed to induce precipitation from 1.5SBF even after 9 days immersion. Incorporation of Nap into Polyactive, however, significantly promoted the calcification of the composites in 1.5SBF. All the composites showed a calcification layer on their surfaces after 3 days immersion in 1.5SBF. Therefore, Nap probably also has the ability to improve bone bonding rates of the composites when implanted in vivo. The strong calcificationinducing capacity of Nap is probably due to the larger specific surface area of the particles and the resulting high $\mathrm{Ca}^{2+}$ and $\mathrm{HPO}_{4}{ }^{2+}$ concentrations due to the dissolution of Nap.

PAA-coated Nap also has the capacity to promote the calcification of the composites. This can be seen from the calcification induced on the surface of $10 \%$ PAA-Nap after 6 days immersion in 1.5SBF. However, the calcification-inducing ability of PAANap seems to be lower than that of Nap, because $10 \%$ PAA-Nap composites only showed calcification after 6 days immersion, while no calcification on $25 \%$ PAA-Nap composites could be observed after 6 days immersion. PAA may affect the dissolution behaviour of the Nap, but it is also a possibility that the calcification rate of PAA-Nap composites was decreased by the formation of dipole complexes between the PEG segments of Polyactive and PAA molecules ${ }^{27,28}$. Where PEG segments have the capacity to chelate calcium ions from the solution by forming a helix structure in aqueous solution ${ }^{35}$, the formation of complexes between PEG and PAA might have decreased this capacity by interfering with the helix conformation of PEG, and thus with the calcification of the composites.

\section{CONCLUSIONS}

Nap has a prominent stiffening effect for Polyactive 70:30 in the dry state. It has a poor stiffening effect for composites in an aqueous environment. Due to the hygroscopic nature and/or formation of aggregates, the wet strength was impaired by the filler in all the composites. PAA coating on Nap had almost no additional effect on the mechanical properties of composites either in the dry state or in an aqueous environment. On the other hand, while Nap has the ability to promote the calcification of composites when incorporated into Polyactive 70:30, PAA coating of Nap had an adverse effect on the calcification of composites, presumably due to the formation of 
complexes between PAA and PEG segments. To reinforce the polymer by Nap, achieving a more homogeneous dispersion of Nap in the polymer matrix and surface modifications to render the powders less hygroscopic appear to be necessary.

\section{ACKNOWLEDGEMENTS}

We thank S. v.d. Meer for her patient and excellent help with the TEM measurements of nano-apatite.

\section{REFERENCES}

1. de Groot, K., Ceramics of calcium phosphate: preparation and properties. In Bioceramics of Calcium Phosphate, ed. K. de Groot. CRC Press, Boca Raton, FL, 1983, pp. 100-114.

2. Bonfield, W., In vivo evaluation of hydroxyapatite reinforced polyethylene composites. In Materials Characteristics vs. In Vivo Behaviour, ed. P. Ducheyne and J. E. Lemnns. New York Arademy of Srience, 1988, p. 173.

3. Tanner, K.E., Doyle, C. and Bonfield, W., The structure of the interface developed between biomaterials and bone. In Clinical Implant Materials; Advances in Biomaterials, Vol. 9. Elsevier Science, Amsterdam, 1990, p. 149.

4. Verheyen, C.C.P.M., de Wijn, J.R., van Blitterswijk, C. A., Rozing, P.M. and de Groot, K., Resorbable hydroxyapatite reinforced poly(L-lactide) composites with bone bonding ability. In Bone-bonding Biomaterials, ed. P. Ducheyne, T. Kokubo and C. A. van Blitterswijk. Reed Healthcare Communications, 1992, pp. 153-171.

5. Labella, R., Braden, M. and Deb, S., Novel hydroxyapatite based dental composites. Biomaterials, 1994, 15, $1197-1200$.

6. Posner, A. S., The mineral of bone. Clin. Orthop. Rel. Res., 1985, 200, 87-99.

7. Ellies, L. G., Carter, J.M., Natiella, J.R., Featherstone, J.D.B. and Nelson, D.G.A., Quantitative analysis of early in vivo tissue response to synthetic apatitic implants. J. Biomed. Mater. Res., 1988, 22, 137-148.

8. Li, Y., Klein, C. P. A. T., de Wijn, J., van de Meer, S. and Groot, K., Shape change and phase transition of needlelike non-stochiometric apatite crystals. J. Mater. Sci.: Mater. Med., 1994, 5, 263-268.

9. Li, Y., de Wijn, J. R., Klein, C. P. A. T., v.d. Meer, S. and de Groot, K., Preparation and characterization of nanograde osteoapatite-like rod crystals. J. Mater. Sci.: Mater. Med., 1994, 5, 252-255.

10. Okada, A. and Usuki, A., The chemistry of polymerclay hybrids. Mater. Sci. Eng., 1995, C3, 109-115.

11. Ozin, G. A., Nanochemistry: synthesis in diminishing dimensions. Adv. Mater., 1992, 4, 612-649.

12. Giannelis, E.P., A new strategy for synthesizing polymer-ceramic nanocomposites. J. Min. Mater. Soc., 1992, 44, 28-30.

13. Lyons, A. M., Nakahara, S., Marcus, M. A., Pearce, E. M. and Waszczak, J.V., Preparation of copper-poly(2vinylpyridine) nanocomposites. J. Phys. Chem. (Washington), 1991, 95, 1098-1105.

14. Moet, A., Akelah, A., Hiltner, A. and Baer, E., Layered silicate/polystyrene nanocomposite. In Proceedings of the 1994 MRS Symposium, San Francisco, CA, 1994, pp. 91-96.

15. Kasemann, R., Schmidt, H.K. and Wintrich, E., New type of a sol-gel-derived inorganic-organic nanocomposite. In Better Ceramics through Chemistry VI,
Proceedings of Materials Research Society, Vol. 346, Pittsburgh, PA, 1994, pp. 915-921.

16. Pinnavaia, T. J., Lan, T.P. and Wang, M., Clay-polymer nanocomposites: polyether and polyamide systems. In Better Ceramics Through Chemistry VI, Proceedings of Materials Research Society, Vol. 346, Pittsburgh, PA, 1994, pp. 81-88.

17. van Blitterswijk, C. A., Bakker, D., Leenders, H. et al., Interfacial reactions leading to bone-bonding with $\mathrm{PEO} / \mathrm{PBT}$ copolymer (Polyactive ${ }^{\mathrm{TM}}$ ). In Bone-bonding Biomaterials, ed. P. Ducheyne, T. Kokubo and C. A. van Blitterswijk. Reed Healthcare Communications, 1992, pp. 153-171.

18. Okumura, M., van Blitterswijk, C. A., Koerten, H. K. Bakker, D., Hesseling, S.D. and de Groot, K., Bone formation process in porous PEO/PBT copolymer (Polyactive): a histological study of ectopic bone formation induced by rat bone marrow cells. In Bonebonding Biomaterials, ed. P. Ducheyne, T. Kokubo and C. A. van Blitterswijk. Reed Healthcare Communications, 1992, pp. 189-200.

19. van Blitterswijk, C. A., van dan Brink, J., Leenders, H. and Bakker, D., The effect of PEO ratio on degradation, ralcification and hone honding of PEO/PBT copolymer (Polyactive). Cells Mater., 1993, 3, 23-26.

20. Gaillard, M.L., van den Brink, J., van Blitterswijk, C. A. and Luklinska, Z.B., Applying a calcium phosphate layer on PEO/PBT copolymers affects bone formation in vivo. J. Mater. Sci.: Mater. Med., 1994, 5, 424-428.

21. Gaillard, M. L. and van Blitterswijk, C. A., Pre-operative addition of calcium ions or calcium phosphate to $\mathrm{PEO} /$ PBT copolymers (Polyactive) stimulates bone mineralization in vitro. J. Mater. Sci.: Mater. Med., 1994, 5, 695-701.

22. Liu, Q., de Wijn, J.R., Bakker, D. and van Blitterswijk, C. A., Surface modification of hydroxyapatite to introduce interfacial bonding with Polyactive ${ }^{\mathrm{TM}} 70 / 30$ in a biodegradable composite. J. Mater. Sci.: Mater. Med., 1996, 7, 551-557.

23. Liu, Q., de Wijn, J. R., van Toledo, M., Bakker, D. and van Blitterswijk, C. A., Polyacids as bonding agents in hydroxyapatite/polyether-ester (Polyactive ${ }^{\text {TM }}$ 30/70 composites). J. Mater. Sci.: Mater. Med. (submitted).

24. Kokubo, T., Kushitani, H., Sakka, S. and Kitsugi, T., Solutions able to reproduce in vivo surface-structure changes in bioactive glass-ceramic A-W. J. Biomed Mater. Res., 1990, 24, 721-734.

25. Skinner, J.C., Prosser, H. J., Scott, R.P. and Wilson A.D., Adsorption of carboxylate cements to hydroxyapatite. I. The effect of the structure of aliphatic carboxylates on their uptake by hydroxyapatite. Biomaterials, 1986, 7, 438-440.

26. Ellis, J., Jackson, A.M., Scott, R.P. and Wilson, A.D. Adhesion of carboxylate cements to hydroxyapatite. III Adsorption of poly(alkenoic acids). Biomaterials, 1990, 11, 379-384.

27. Bailey, F.E. and Koleske, J. V., Association complexes of poly(ethylene oxide). In Poly(ethylene Oxide). Academic Press, New York, 1976, Chapter 5.

28. Liu, Q., de Wijn, J.R. and van Blitterswijk, C.A. Intermolecular complexation between PEG/PBT block copolymer and polyelectrolytes polyacrylic acid and maleic acid copolymer. Eur. Polym. I. (accepted).

29. Soltesz, U., Ceramics in composites: review and current status. In Bioceramics: Materials Characteristics Versus In Vivo Behaviour, Annals of the New York Academy of Sciences, Vol. 523, ed. P. Ducheyne and J.E. Lemons. 1988, pp. 137-156.

30. Giannelis, E. P., Polymer layered silicate nanocomposites. Adv. Mater., 1996, 8, 29-35. 
31. Shi, H., Lan, T. and Pinnavaia, T. J., Interfacial effects on the reinforcement properties of polymer-organoclay nanocomposites. Chem. Mater., 1996, 8, 1584-1587.

32. Radder, A. M., Davies, J.E., Leenders, H., v.d. Meer, S. and van Blitterswijk, C. A., Post-operative carbonateapatite formation in a polymer matrix: characterization and relation to bone-bonding. Bioceramics, 1993, 6, 345-351.

33. Radder, A.M. and van Blitterswijk, C. A., Abundant post-operative calcification of an elastomer matrix calcium phosphate-polymer composite for bone reconstruction: a preliminary study. J. Mater. Sci.: Mater. Med., 1993, 5, 320-325.

34. Radder, A.M., Davies, J.E., Leenders, H. and van Blitterswijk, C. A., Interfacial behaviour of PEC/PBT copolymer (Polyactive ${ }^{\mathrm{TM}}$ ) in a calvarial system: an in vitro study. J. Biomed. Mater. Res., 1994, 28, 269-277.

35. Thoma, R. J., Poly(ether)urethane reactivity with metalion in calcification and environmental stress cracking. J. Biomater. Applic., 1987, 1, 449-486. 